\title{
Fabrication of fluorescent nanoparticles of dendronized perylenediimide by laser ablation in water
}

\author{
R. Yasukuni $\cdot$ T. Asahi $\cdot$ T. Sugiyama $\cdot$ H. Masuhara $\cdot$ \\ M. Sliwa $\cdot$ J. Hofkens $\cdot$ F.C. De Schryver $\cdot$ M. Van \\ der Auweraer • A. Herrmann • K. Müllen
}

Received: 12 October 2007 / Accepted: 9 April 2008 / Published online: 6 June 2008

(c) Springer-Verlag 2008

\begin{abstract}
Highly fluorescent organic nanoparticles with size of about $300 \mathrm{~nm}$ were prepared by nanosecond laser ablation of micrometer-sized powder of dendronized perylenediimide dispersed in water. The nanoparticle colloidal solution provided a fluorescence quantum yield of 0.58 . The absorption and emission spectral studies demonstrated that the bulky dendron groups at the side bays of perylenediimide
\end{abstract}

R. Yasukuni · T. Asahi $(\bowtie)$

Department of Applied Physics, Osaka University,

2-1 Yamadaoka, Suita, Osaka 565-0871, Japan

e-mail: asahi@ap.eng.osaka-u.ac.jp

T. Sugiyama $\cdot$ H. Masuhara

Graduate School of Materials Science, Nara Institute of Science

and Technology, Ikoma, Nara 630-0192, Japan

\section{H. Masuhara}

Department of Applied Chemistry and Institute of Molecular Science, National Chiao Tung University, Hsinchu 30010, Taiwan

M. Sliwa - J. Hofkens - F.C. De Schryver - M. Van der Auweraer Department of Chemistry and Institute for Nanoscale Physics and Chemistry (INPAC), Katholieke Universiteit Leuven,

Celestijnenlaan 200F, 3001 Heverlee, Belgium

\section{A. Herrmann}

Department of Polymer Chemistry, Zernike Institute for

Advanced Materials, University of Groningen, Nijenborgh 4, 9747 AG Groningen, The Netherlands

\section{K. Müllen}

Max-Planck-Institut für Polymerforschung, Ackermannweg 10, 55128 Mainz, Germany

Present address:

M. Sliwa

LASIR, UMR 8516, CNRS, Université des Sciences et Technologies de Lille, Bât C5, 59655 Villeneuve d'Ascq Cedex, France chromophore efficiently suppress the interchromophoric interactions in the nanoparticles. Fluorescence measurement on several single nanoparticles underlines that the prepared nanoparticles are bright and photo-stable enough to be a useful probe for single particle fluorescence investigation.

PACS 52.38.Mf $\cdot$ 61.46.Hk $\cdot 87.64 . \mathrm{Kv}$

\section{Introduction}

Luminescent nanoparticles composed of organic molecules have attracted considerable interest since they show sizedependent fluorescent properties different from bulk solid samples [1-6]. In recent years, they have been investigated for various potential applications, such as biological labels [7, 8], chemical sensors [9, 10], and light emitting diodes [11]. In comparison with molecules, organic nanoparticles are expected to show high photostability and efficient fluorescence in various media. These are essentially important in fluorescent labeling applications, such as fluorescence bioimaging and single molecular spectroscopy. For example, single molecules of most commercial dyes undergo photo-bleaching in a few milliseconds under typical excitation conditions using a laser fluorescence microscope. On the contrary, because large numbers of chromophores are incorporated, single nanoparticles show bright fluorescence even at a low excitation power, do not undergo rapid photo-bleaching and give less emission blinking and off states which are generally observed in single molecule experiment [12].

Recently, we have succeeded in preparing various organic nanoparticles using phthalocyanines, other pigments, and fullerene by laser ablation of their microcrystals in water [13-17]. This method is really interesting because 
a suspension of a target bulk material directly converts into its colloidal solution without any organic solvents. In this paper, we have extended this method to fluorescent organic nanoparticles using a perylenediimide (PDI) compound. This is one of the most important classes of fluorescent dyes having a fluorescence quantum yield near one and a high photostability. However, the fabrication of highly fluorescent PDI nanoparticles is not simple, because molecular aggregation leads to a fluorescence quenching and/or excimer emission due to intermolecular interactions. PDI nanoparticles were recently prepared by a reprecipitation method using $N, N^{\prime}$-bis(2,5-di-tertbutylphenyl)-3,4,9,10-perylenedicarboxyimide and $N, N^{\prime}$ bis(2,6-dimethylphenyl)-3,4,9,10-perylenedicarboxyimide, but their emission spectra were characteristic of a strong intermolecular interaction as an excimer-like emission and a low fluorescence quantum yield was observed [18, 19]. To suppress this intermolecular interaction, we here used a bay substituted dendronized PDI (DPDI) [20], where a bulky dendron group at the PDI chromophore bays should hinder the electronic interactions among different PDI chromophores. Moreover, bay substituted PDI single molecules resist photo-bleaching for a few minutes under a common excitation light through a microscope at excitation power of a $\mathrm{kW} / \mathrm{cm}^{2}$ order $[21,22]$. With our laser ablation by $532 \mathrm{~nm}$ nanosecond laser irradiation, we prepared for the first time highly fluorescent PDI nanoparticles dispersed in water. The absorption and emission spectra confirm that the bulky dendron group efficiently suppresses the electronic interactions among PDI chromophores in the nanoparticles and that the colloidal solution with nanoparticles exhibits bright fluorescence with a quantum yield of 0.58 . We also present the results of single particle fluorescence experiment and demonstrate that the bay substituted DPDI nanoparticle is considered to be a useful probe for single particle spectroscopy.

\section{Experimental}

The synthesis of DPDI has been described previously [20], and its nanoparticles were prepared by laser ablation in water $[14,15]$. The powder was added to an aqueous solution $\left(3 \times 10^{-3} \mathrm{wt} \%\right)$ containing $8 \mathrm{mM}$ of sodium dodecyl sulfate (Wako), then the suspension was sonicated for 30 minutes. The mixture $(3.0 \mathrm{~mL})$ was put into a $1 \mathrm{~cm} \times 1 \mathrm{~cm} \times 5 \mathrm{~cm}$ quartz cuvette, stirred vigorously with a magnetic stirrer, and then simultaneously exposed to the second harmonic of a nanosecond YAG laser (Continuum, Surelite II, $532 \mathrm{~nm}$, $10 \mathrm{~Hz}, 8 \mathrm{~ns}$ FWHM). The spot area was approximately $22 \mathrm{~mm}^{2}$, and the laser intensity was adjusted using a polarizer.

The size of nanoparticles was estimated by dynamic light scattering (DLS) measurement (Ohtsuka-denshi, DLS$70 \mathrm{H})$. The UV-VIS extinction and emission spectra of the nanoparticle colloidal solution were measured using a UV-VIS-NIR scanning spectrophotometer (Shimadzu UV3100PC) and a fluorescence spectrophotometer (Hitachi F4500), respectively. The scattering spectra were obtained by measuring the side scattering light with the same fluorescence spectrophotometer, where the excitation and observation wavelengths are scanned synchronously. Fluorescence measurement of single nanoparticles was performed using DPDI nanoparticles dispersed in a thin polyvinyl alcohol (PVA) film. Sample films were prepared by spincoating a mixture (1:1) of the nanoparticle colloidal solution and an aqueous PVA solution (10 wt $\%, M_{w} ; 88000$, Wako) on cleaned glass cover slips (Micro-Slide Glass, MATSUNAMI). The sample was set on an inverted confocal scanning fluorescence microscope, and time-resolved measurements on individual nanoparticles were carried out using the time-correlated single photon counting (TCSPC) technique. The excitation pulse at $543 \mathrm{~nm}(8.18 \mathrm{MHz}, 1.2 \mathrm{ps}$ FWHM) was obtained from the frequency doubled output of an optical parametric oscillator (GWU) pumped by a Ti:Sapphire laser (Tsunami, Spectra Physics). The detailed descriptions of the setup and the data acquisition process have been published previously [22, 23]. In short, the excitation light was rendered circularly polarized by a Berek compensator, directed into an inverted microscope (Olympus IX 70) and focused onto the sample through an oil immersion objective $(1.3 \mathrm{NA}, \times 100$, Olympus). The fluorescence was collected through the same objective and focused onto an avalanche photodiode (SPCM 15, EG\&G). Timeresolved data were collected with TCSPC card (SPC 630, Becker \& Hickl) operated in first-in-first-out mode.

\section{Results and discussion}

\subsection{Nanoparticles formation}

The initial powder sank to the bottom of the cuvette before the laser irradiation and the supernatant was almost colorless, which means that DPDI molecules are hardly dissolved in water. After being exposed to laser pulses at laser fluences above the threshold for $10 \mathrm{~min}$, the suspension shows a magenta color. The apparent absorption spectra of the supernatants obtained after leaving the solution to settle for $1 \mathrm{hr}$ are shown in Fig. 1a. The spectra at laser fluences above $30 \mathrm{~mJ} / \mathrm{cm}^{2}$ have peaks similar to those of DPDI molecules in acetone, and they exhibit a long wavelength tail due to scattering. These spectral features are characteristics of the nanoparticle colloidal solution, as discussed later. The fluence dependence on the absorbance at the peak around $580 \mathrm{~nm}$ in Fig. 1b clearly demonstrates that the nanoparticle formation requires a high photon density as in the case of laser ablation of other organic dyes in water. These results 


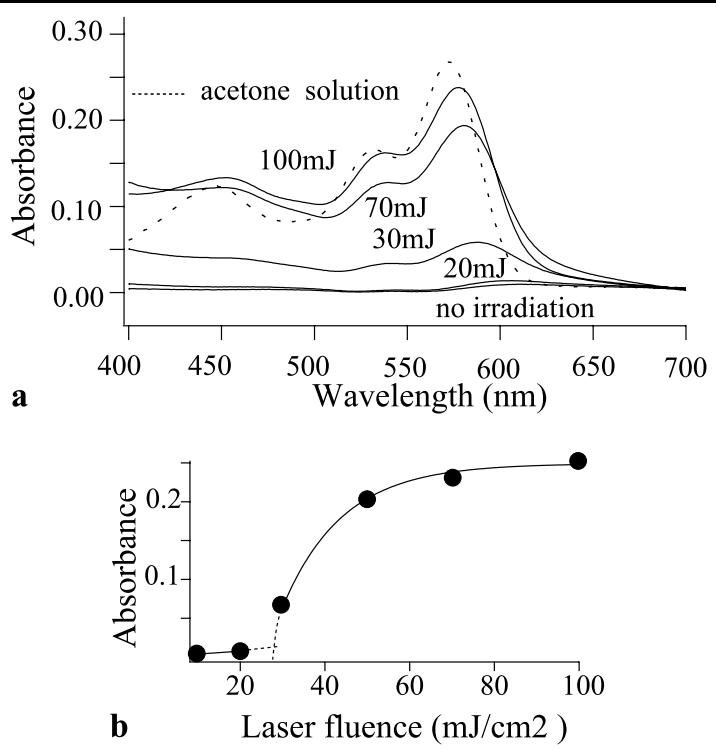

Fig. 1 (a) Absorption spectra of a suspension of DPDI in water (solid lines) before the laser irradiation and after 10 minutes laser irradiation at laser fluences of $20,30,70,100 \mathrm{~mJ} / \mathrm{cm}^{2}$, and absorption spectrum of $0.01 \mathrm{mM}$ acetone solution of DPDI (dashed line). (b) Laser fluence dependence on the peak absorbance of the supernatant after leaving the solution to settle for $1 \mathrm{hr}$

suggest that DPDI is dispersed in water as nanoparticles and that laser ablation of the micrometer-sized powder is responsible for the nanoparticle formation in water [13-16]. At higher laser fluences, the colloidal solution of prepared DPDI nanoparticles was stable, and its absorption spectrum did not show any significant change even after leaving the solution to settle for one day.

\subsection{Absorption and fluorescence spectra of colloidal solution}

As shown in Fig. 1a, the absorption spectra of the DPDI nanoparticles colloidal solutions exhibit a red-shifted peak compared to that of the acetone solution and show a gradual tail in the wavelength region longer than $630 \mathrm{~nm}$. It should be, however, noted that conventional absorption measurement of colloid samples does not provide an absorption spectrum of the nanoparticles but an extinction spectrum. In general, the extinction cross section corresponds to a sum of optical absorption and scattering cross sections, and the scattering contribution increases with the particle size. Therefore, it can be considered that the scattering loss is the main reason of the discrepancy in the absorption spectra between the nanoparticle colloidal solution and the molecules.

In order to characterize precisely the spectroscopic properties of DPDI nanoparticles, we prepared the nanoparticles by the laser irradiation at laser fluence of $60 \mathrm{~mJ} / \mathrm{cm}^{2}$ for $50 \mathrm{~min}$ and measured the extinction and scattering spectra of the nanoparticle colloidal solution with a mean size of

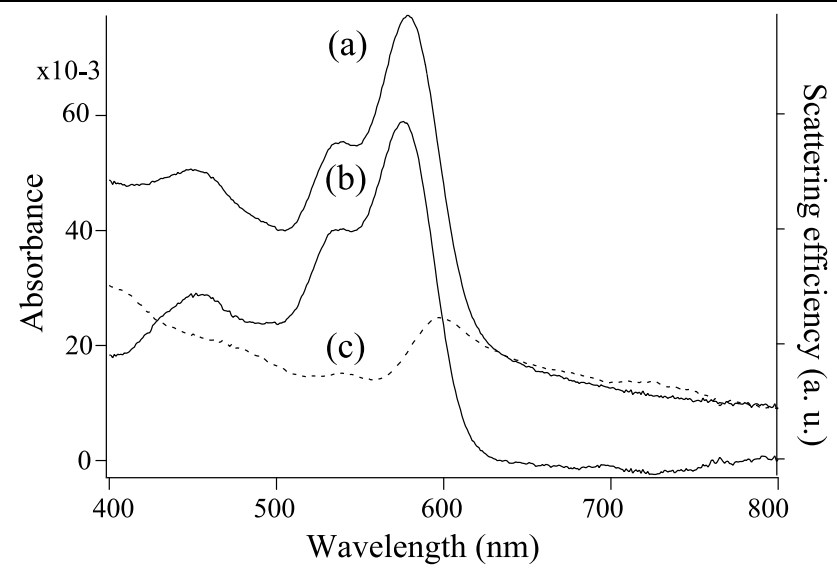

Fig. 2 Extinction (a), absorption (b) and scattering (c) spectra of DPDI nanoparticles colloidal solution after laser irradiation at $60 \mathrm{~mJ} / \mathrm{cm}^{2}$ for $50 \mathrm{~min}$. The absorption spectrum was calculated by subtracting the scattering spectrum from the extinction one after normalizing them at the wavelength of $800 \mathrm{~nm}$

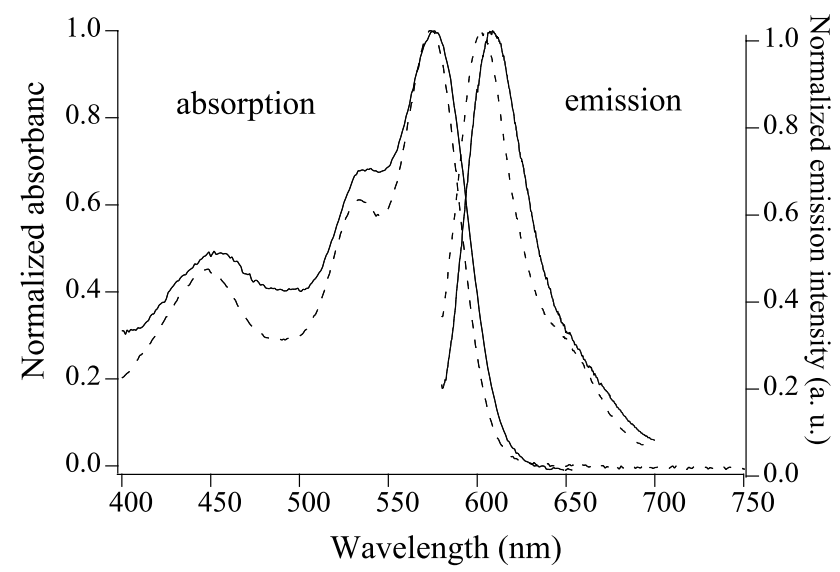

Fig. 3 Normalized absorption and emission spectra at $570 \mathrm{~nm}$ excitation wavelength of DPDI nanoparticles colloidal (solid lines) and $2 \mu \mathrm{M}$ acetone solution (dashed lines). Each spectrum was normalized at the peak wavelength

$300 \mathrm{~nm}$ as determined by DLS. The results are shown in Fig. 2, where the extinction spectrum is obtained by the conventional absorption measurement, and the absorption spectrum is calculated by subtracting the scattering spectrum from the extinction one after normalizing them at $800 \mathrm{~nm}$, a wavelength where the nanoparticle shows no absorption. We can ascribe the tail in the extinction spectrum to the light scattering of the nanoparticles. Furthermore, the obtained "true" absorption spectrum of the nanoparticles shows that absorption maxima coincide with those of the molecules dissolve in acetone as shown in Fig. 3. This means that the intermolecular interactions among PDI chromophores are very weak in the nanoparticles.

The very weak interchromophoric interaction is also confirmed by the fluorescence measurements. Figure 3 demonstrates that the fluorescence spectrum of the nanoparticle 
Fig. 4 (a) Fluorescence image of DPDI nanoparticles dispersed in a PVA film at $540 \mathrm{~nm}$ excitation wavelength.

(b) Normalized fluorescence decay curves of three different DPDI single nanoparticles in PVA (solid lines) and a single DPDI molecule in PMMA (dash line) at $543 \mathrm{~nm}$ excitation wavelength

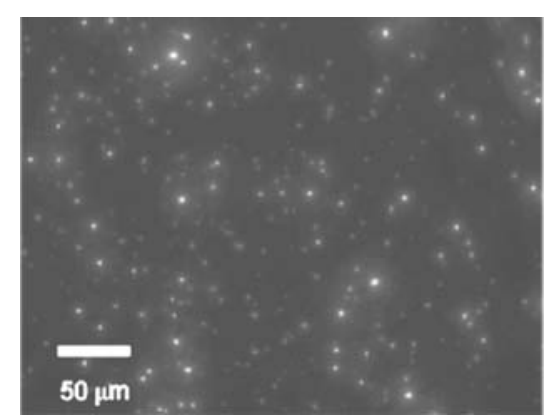

$\mathbf{a}$

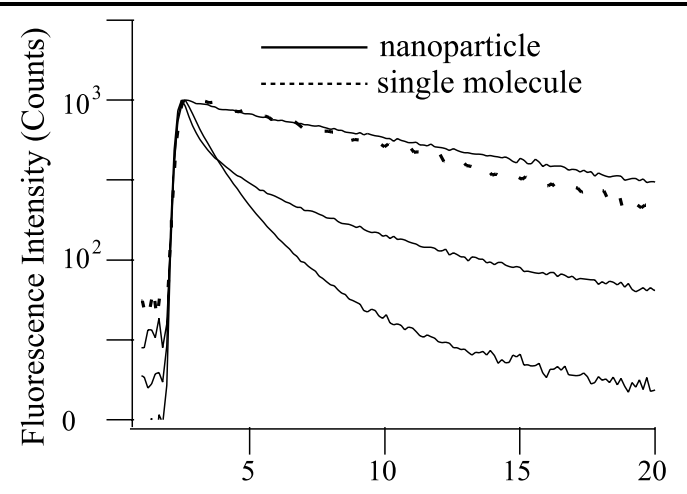

b

Time (ns) colloidal solution is similar to that of the acetone solution and does not give an excimer emission band. The fluorescence peak energy is lower by $100 \mathrm{~cm}^{-1}$ than that of the molecular solution. However, this will be mainly due to reabsorption of fluorescence by other chromophores in the same nanoparticle as the absorption and emission spectra overlap each other near the peak of the emission. The fluorescence quantum yield of the nanoparticles was estimated to be 0.58 by comparing the fluorescence intensity of the diluted colloidal solution to that of the acetone solution and by assuming that the quantum yield of the acetone solution equals one [20]. Although the obtained quantum yield of the nanoparticles is high, it is still smaller than unity suggesting that nonradiative deactivation channels of the excited single state, such as quenching at the surface or by impurities or packing defects, should be considered.

\subsection{Single particle fluorescence measurement}

Figure 4a is a typical fluorescence image of a nanoparticlesdoped PVA film under a conventional fluorescent microscope at $540 \mathrm{~nm}$ excitation wavelength. This image shows quite a big scatter in the intensity of fluorescence spots, which is due to a large distribution in the particle size. Using this sample film, the spectroscopic investigation of single nanoparticles was achieved with a scanning confocal fluorescence microscope. 48 fluorescence spots with the size about $300 \mathrm{~nm}$ (the lateral resolution limited by the light diffraction) were selected, and the fluorescence decay of their emission was measured by the time-correlation single photon counting technique. As the size corresponds to the DLS's one, each spot can be considered to be the fluorescence from an individual single nanoparticle. Figure $4 \mathrm{~b}$ demonstrates the decay profiles for three single nanoparticles as representative examples and that of a single DPDI molecule. The fluorescence decay of single molecules in a PMMA film could be fitted by a mono-exponential function, and the fluorescence decay time was about $5 \mathrm{~ns}$. On the contrary, fluorescence decay profiles of the single nanoparticles differ from particle to particle, and most of them contain at least two exponential components. The shorter decay time component indicates fluorescence quenching in the nanoparticles. More detailed analysis on the single particle spectroscopic data is now under progress and will be reported elsewhere.

It is notable that the excitation intensity for observing nanoparticles was much lower than that for single molecule detection, $\sim 10 \mathrm{~W} / \mathrm{cm}^{2}$ and $1 \mathrm{~kW} / \mathrm{cm}^{2}$ at the sample, respectively, in order to collect the same number of fluorescence photons (about 30000 counts/s). Thus, even by using only a few tens of $\mathrm{W} / \mathrm{cm}^{2}$ for the excitation intensity, the fluorescence intensity was higher than that of a single molecule. Moreover, many nanoparticles showed less emissionoff states in the time traces of photon count, which suggests that the DPDI nanoparticles can be considered as a stable photon source. Therefore it can be concluded that the DPDI nanoparticles prepared by laser ablation in water are bright and photo-stable enough for single particle fluorescence investigation. The size and morphology dependence of the fluorescence dynamics can be examined by fabricating nanoparticles with various sizes and different morphologies by tuning the laser irradiation condition, such as laser intensity and pulse width $[15,16]$.

\section{Conclusion}

We have successfully prepared DPDI nanoparticles by the laser ablation in water, and demonstrated that the prepared colloidal solution showed a high fluorescence quantum yield of 0.58 . The fluorescence and absorption spectra of the nanoparticles are in good agreement with those of DPDI molecules in the acetone solution, which means that electronic interactions between PDI chromophores in the nanoparticles are very weak due to the bulky dendron group. A single particle fluorescence experiment by confocal laser fluorescence microscopy suggested that the DPDI nanoparticles are very useful and applicable to fluorescence imaging 
probes. It was demonstrated that the laser ablation in water is a very useful technique to fabricate organic nanoparticles of fluorescent organic materials as well as other various organic ones.

Acknowledgements This work was supported in part by a Grantin-Aid for Scientific Research in a Priority Area "Strong PhotonsMolecules Coupling Fields" (area 470, No. 19049011) from Ministry of Education, Culture, Sports, Science, and Technology of Japan and, as a part of the European Science Foundation EUROCORES Programme SONS, was also supported from funds by the FWO and the EC Sixth Framework Programme, under contract No. ERAS-CT-2003980409. Support from the FWO (grant G.0366.06), the KULeuven Research Fund (GOA 2006/2), the Flemish Ministry of Education (ZWAP 04/007) and the Federal Science Policy of Belgium (IAP VI/27), and the Deutsche Forschungsgemeinschaft (SFB 625) and BASF AG is gratefully acknowledged.

\section{References}

1. H. Oikawa, T. Mitsui, T. Onodera, H. Kasai, H. Nakanishi, T. Sekiguchi, Jpn. J. Appl. Phys. 42, L111 (2003)

2. T. Onodera, H. Kasai, S. Okada, H. Oikawa, K. Mizuno, M. Fujitsuka, O. Ito, H. Nakanishi, Opt. Mater. 21, 595 (2003)

3. S. Li, L. He, F. Xiong, Y. Li, G. Yang, J. Phys. Chem. B 108, 10887 (2004)

4. B.K. An, S.K. Kwon, S.D. Jung, S.Y. Park, J. Am. Chem. Soc. 124, 14410 (2002)

5. S.J. Lim, B.K. An, S.D. Jung, M.A. Chung, S.Y. Park, Angew. Chem. Int. Ed. 43, 6346 (2004)

6. H. Matsune, T. Asahi, H. Masuhara, H. Kasai, H. Nakanishi, Pol. J. Chem. 4, 687 (2008)

7. Y. Zhou, G. Bian, L. Wang, L. Dong, L. Wang, J. Kan, Spectrochim. Acta Part A 61, 1841 (2005)
8. L. Wang, T. Xia, L. Wang, H. Chun, L. Dong, G. Bian, Microchem. Acta 149, 267 (2005)

9. L. Wang, L. Dong, G.R. Bian, L.Y. Wang, T.T. Xia, H.Q. Chen, Anal. Bioanal. Chem. 382, 1300 (2005)

10. R. Jagannathan, G.C. Irvin Jr., Adv. Funct. Mater. 15, 1501 (2005)

11. R. Jagannathan, G. Irvin, T. Blanton, S. Jagannathan, Adv. Funct. Mater. 16, 747 (2006)

12. J.N. Clifford, T.D.M. Bell, P. Tinnefeld, M. Heilemann, S.M. Melnikov, J. Hotta, M. Sliwa, P. Dedecker, M. Sauer, J. Hofkens, E.K.L. Yeow, J. Phys. Chem. B 111, 6987 (2007)

13. Y. Tamaki, T. Asahi, H. Masuhara, J. Phys. Chem. A 106, 2135 (2002)

14. Y. Tamaki, T. Asahi, H. Masuhara, Jpn. J. Appl. Phys. 42, 2725 (2003)

15. T. Sugiyama, T. Asahi, H. Takeuchi, H. Masuhara, Jpn. J. Appl. Phys. 45, 384 (2006)

16. T. Sugiyama, T. Asahi, H. Masuhara, Chem. Lett. 33, 724 (2004)

17. H.-G. John, S. Ryo, T. Sugiyama, I. Oh, H. Masuhara, T. Asahi, Chem. Lett. 36, 1160 (2007)

18. J. Gesquiere, T. Uwada, T. Asahi, H. Masuhara, P.F. Barbara, Nano Lett. 5, 1321 (2005)

19. S. Masuo, A. Masuhara, T. Akashi, M. Muranushi, S. Machida, H. Kasai, H. Nakanishi, H. Oikawa, A. Itaya, Jpn. J. Appl. Phys. 46, L268 (2007)

20. J.Q. Qu, N.G. Pschirer, D.J. Liu, A. Stefan, F.C. De Schryver, K. Müllen, Chem. Eur. J. 10, 528 (2004)

21. H. Uji-i, S.M. Melnikov, A. Deres, G. Bergamini, F.C. De Schryver, A. Herrmann, K. Müllen, J. Enderlein, J. Hofkens, Polymer 47, 2511 (2006)

22. C. Flors, I. Oesterling, T. Schnitzler, E. Fron, G. Schweitzer, M. Sliwa, A. Herrmann, M. Van der Auweraer, F.C. De Schryver, K. Müllen, J. Hofkens, J. Phys. Chem. C 111, 4861 (2007)

23. M. Sliwa, C. Flors, I. Oesterling, J. Hotta, K. Müllen, F.C. De Schryver, J. Hofkens, J. Phys.: Condens. Matter 19, 445004 (2007) 